\title{
Clinical Utility of Methicillin-Resistant Staphylococcus aureus Polymerase Chain Reaction Nasal Swab Testing in Lower Respiratory Tract Infections
}

Caitlin Caulfield, PharmD, Kristin Linder, PharmD, BCIDP, Kelly Shepard, PharmD, David O'Sullivan, $P h D$, and Casey Dempsey, PharmD, BCIDP

\section{ABSTRACT}

Objective: To assess the utility of methicillin-resistant Staphylococcus aureus (MRSA) polymerase chain reaction (PCR) nasal swab testing in patients with lower respiratory tract infections (LRTI).

Design and setting: Multicenter, retrospective, electronic chart review conducted within the Hartford HealthCare system.

Participants: Patients who were treated for LRTIs at the Hospital of Central Connecticut or Hartford Hospital between July 1, 2018, and June 30, 2019.

Measurements: The primary outcome was anti-MRSA days of therapy (DOT) in patients who underwent MRSA PCR testing versus those who did not. In a subgroup analysis, we compared anti-MRSA DOT among patients with appropriate versus inappropriate utilization of the MRSA PCR test.
Results: Of the 319 patients treated for LRTls, 155 (48.6\%) had a MRSA PCR ordered, and appropriate utilization occurred in $94(60.6 \%)$ of these patients. Anti-MRSA DOT in the MRSA PCR group $(n=155)$ was shorter than in the group that did not undergo MRSA PCR testing $(\mathrm{n}=164)$, but this difference did not reach statistical significance (1.68 days [interquartile range $\{\mathrm{IQR}\}, 0.80-2.74]$ vs 1.86 days [IQR, $0.56-3.33$ ], $P=0.458)$. In the subgroup analysis, anti-MRSA DOT was significantly shorter in the MRSA PCR group with appropriate utilization compared to the inappropriate utilization group (1.16 [IQR, 0.44-1.88] vs 2.68 [IQR, 1.75-3.76], $P<0.001)$.

Conclusion: Appropriate utilization of MRSA PCR nasal swab testing can reduce DOT in patients with LRTI. Further education is necessary to expand the appropriate use of the MRSA PCR test across our health system.

Keywords: MRSA; LRTI; pneumonia; antimicrobial stewardship; antibiotic resistance.
M ore than 300,000 patients were hospitalized with methicillin-resistant Staphylococcus aureus (MRSA) infections in the United States in 2017, and at least 10,000 of these cases resulted in mortality. ${ }^{1}$ While MRSA infections overall are decreasing, it is crucial to continue to employ antimicrobial stewardship tactics to keep these infections at bay. Recently, strains of $S$. aureus have become resistant to vancomycin, making this bacterium even more difficult to treat. ${ }^{2}$

A novel tactic in antimicrobial stewardship involves the use of MRSA polymerase chain reaction (PCR) nasal swab testing to rule out the presence of MRSA in patients with lower respiratory tract infections (LRTI).
If used appropriately, this approach may decrease the number of days patients are treated with anti-MRSA antimicrobials. Waiting for cultures to speciate can take up to 72 hours, ${ }^{3}$ meaning that patients may be exposed to 3 days of unnecessary broad-spectrum antibiotics. Results of MRSA PCR assay of nasal swab specimens can be available in 1 to 2 hours, ${ }^{4}$ allowing for more rapid de-escalation of therapy. Numerous studies have shown

From the Hospital of Central Connecticut, New Britain, CT (Dr. Caulfield and Dr. Shepard); Hartford Hospital, Hartford, CT (Dr. Linder and Dr. Dempsey); and the Hartford HealthCare Research Program, Hartford, CT (Dr. O'Sullivan). 
that this test has a negative predictive value (NPV) greater than 95\%, indicating that a negative nasal swab result may be useful to guide de-escalation of antibiotic therapy. ${ }^{5-8}$ The purpose of this study was to assess the utility of MRSA PCR nasal swab testing in patients with LRTI throughout the Hartford HealthCare system.

\section{Methods \\ Design}

This study was a multicenter, retrospective, electronic chart review. It was approved by the Hartford HealthCare Institutional Review Board (HHC-2019-0169).

\section{Selection of Participants}

Patients were identified through electronic medical record reports based on ICD-10 codes. Records were categorized into 2 groups: patients who received a MRSA PCR nasal swab testing and patients who did not. Patients who received the MRSA PCR were further categorized by appropriate or inappropriate utilization. Appropriate utilization of the MRSA PCR was defined as MRSA PCR ordered within 48 hours of a new vancomycin or linezolid order, and anti-MRSA therapy discontinued within 24 hours of a negative result. Inappropriate utilization of the MRSA PCR was defined as MRSA PCR ordered more than 48 hours after a new vancomycin or linezolid order, or continuation of anti-MRSA therapy despite a negative MRSA PCR and no other evidence of a MRSA infection.

Patients were included if they met all of the following criteria: age 18 years or older, with no upper age limit; treated for a LRTI, identified by ICD-10 codes (J13-22, J44, J85); treated with empiric antibiotics active against MRSA, specifically vancomycin or linezolid; and treated at the Hospital of Central Connecticut (HOCC) or Hartford Hospital $(\mathrm{HH})$ between July 1, 2018, and June 30, 2019, inclusive. Patients were excluded if they met 1 or more of the following criteria: age less than 18 years old; admitted for 48 hours or fewer or discharged from the emergency department; not treated at either facility; treated before July 1, 2018, or after June 30, 2019; treated for ventilator-associated pneumonia; received anti-MRSA therapy within 30 days prior to admission; or treated for a concurrent bacterial infection requiring anti-MRSA therapy.

\section{Outcome Measures}

The primary outcome was anti-MRSA days of therapy (DOT) in patients who underwent MRSA PCR testing compared to patients who did not undergo MRSA PCR testing. A subgroup analysis was completed to compare anti-MRSA DOT within patients in the MRSA PCR group. Patients in the subgroup were categorized by appropriate or inappropriate utilization, and anti-MRSA DOT were compared between these groups. Secondary outcomes that were evaluated included length of stay (LOS), 30-day readmission rate, and incidence of acute kidney injury (AKI). Thirty-day readmission was defined as admission to $\mathrm{HOCC}, \mathrm{HH}$, or any institution within Hartford HealthCare within 30 days of discharge. AKI was defined as an increase in serum creatinine by $\geq 0.3$ $\mathrm{mg} / \mathrm{dL}$ in 48 hours, increase $\geq 1.5$ times baseline, or a urine volume $<0.5 \mathrm{~mL} / \mathrm{kg} / \mathrm{hr}$ for 6 hours.

\section{Statistical Analyses}

The study was powered for the primary outcome, antiMRSA DOT, with a clinically meaningful difference of 1 day. Group sample sizes of 240 in the MRSA PCR group and 160 in the no MRSA PCR group would have afforded $92 \%$ power to detect that difference, if the null hypothesis was that both group means were 4 days and the alternative hypothesis was that the mean of the MRSA PCR group was 3 days, with estimated group standard deviations of $80 \%$ of each mean. This estimate used an alpha level of 0.05 with a 2-sided t-test. Among those who received a MRSA PCR test, a clinically meaningful difference between appropriate and inappropriate utilization was $5 \%$.

Descriptive statistics were provided for all variables as a function of the individual hospital and for the combined data set. Continuous data were summarized with means and standard deviations (SD), or with median and interquartile ranges (IQR), depending on distribution. Categorical variables were reported as frequencies, using percentages. All data were evaluated for normality of distribution. Inferential statistics comprised a Student's t-test to compare normally distributed, continuous data between groups. Nonparametric distributions were compared using a Mann-Whitney $U$ test. Categorical comparisons were made using a Fisher's 


\section{Table 1. Baseline Characteristics: MRSA PCR vs No MRSA PCR Testing}

\begin{tabular}{|c|c|c|c|}
\hline Characteristic & $\begin{array}{l}\text { No MRSA PCR } \\
(n=164)\end{array}$ & $\begin{array}{c}\text { MRSA PCR } \\
(n=155)\end{array}$ & $P$ Value \\
\hline Age, mean (SD), yr & $69.90(17.23)$ & $68.50(17.57)$ & 0.473 \\
\hline Race, no. (\%) & & & 0.370 \\
\hline White & $118(72.0)$ & $103(66.9)$ & \\
\hline Black & $12(7.3)$ & 19 (12.3) & \\
\hline Asian & $3(1.8)$ & $3(1.9)$ & \\
\hline Hispanic & $26(15.9)$ & $20(13.0)$ & \\
\hline Other & $5(3.0)$ & $9(5.8)$ & \\
\hline Sex, no. (\%) & & & 0.910 \\
\hline Female & $71(43.3)$ & $69(44.5)$ & \\
\hline Male & $93(56.7)$ & $86(55.5)$ & \\
\hline Weight, mean (SD), kg & $82.92(29.74)$ & $79.28(21.83)$ & 0.213 \\
\hline Body mass index, mean (SD) & $29.36(9.15)$ & $28.29(7.17)$ & 0.247 \\
\hline Hospital, no. (\%) & & & 0.175 \\
\hline Hartford Hospital & $134(81.7)$ & $117(75.5)$ & \\
\hline Hospital of Central Connecticut & $30(18.3)$ & $38(24.5)$ & \\
\hline Charlson Comorbidity Index, median (IQR) & $5.0(3.0-6.0)$ & $5.0(3.0-7.0)$ & 0.439 \\
\hline Type of pneumonia, no. (\%) & & & 0.749 \\
\hline CAP & 56 (34.1) & $47(30.3)$ & \\
\hline CAP with risk factors & $95(57.9)$ & $96(61.9)$ & \\
\hline Hospital-acquired pneumonia & $13(7.9)$ & $12(7.7)$ & \\
\hline Intensive care unit admission, no. (\%) & $13(7.9)$ & $35(22.6)$ & $<0.001$ \\
\hline Medical/surgical admission, no. (\%) & $150(91.5)$ & $120(77.4)$ & 0.001 \\
\hline \multicolumn{4}{|l|}{ Anti-MRSA agent, no. (\%) } \\
\hline Vancomycin & $164(100)$ & $154(99.4)$ & \\
\hline Linezolid & $0(0)$ & $1(0.6)$ & \\
\hline Chest radiograph positive, no. (\%) & $121(74.2)$ & $131(85.1)$ & 0.017 \\
\hline Infectious diseases consult, no. (\%) & $44(26.8)$ & $55(35.5)$ & 0.095 \\
\hline Pulmonary consult, no. (\%) & $32(19.5)$ & $53(34.2)$ & 0.003 \\
\hline Sepsis at time of anti-MRSA initiation, no. (\%) & $48(29.3)$ & $65(41.9)$ & 0.018 \\
\hline Vasopressors at time of anti-MRSA, no. (\%) & $5(3.0)$ & $6(3.9)$ & 0.688 \\
\hline $\mathrm{SC}$ at time of anti-MRSA initiation, median (IQR), mg/dL & $0.90(0.70-1.32)$ & $1.00(0.78-1.50)$ & 0.470 \\
\hline SC at time of anti-MRSA discontinuation, median (IQR), mg/dL & $0.90(0.70-1.30)$ & $0.90(0.70-1.40)$ & 0.711 \\
\hline SC $48 \mathrm{hr}$ after anti-MRSA discontinuation, median (IQR), mg/dL & $0.90(0.70-1.32)$ & $0.80(0.70-1.35)$ & 0.476 \\
\hline No. of nephrotoxic medications, excluding vancomycin, mean (SD) & $2.38(1.34)$ & $2.48(1.22)$ & 0.489 \\
\hline Use of intravenous contrast, no. (\%) & $56(34.1)$ & $64(41.3)$ & 0.188 \\
\hline \multicolumn{4}{|l|}{ Other antibiotics used, no. (\%) } \\
\hline Azithromycin & $59(36.0)$ & $63(40.6)$ & 0.391 \\
\hline Carbapenems & $1(0.6)$ & $6(3.9)$ & 0.047 \\
\hline Cefepime & $129(78.7)$ & $138(89.0)$ & 0.012 \\
\hline Ceftriaxone & $75(45.7)$ & $73(47.1)$ & 0.807 \\
\hline Piperacillin/tazobactam & $13(7.9)$ & $16(10.3)$ & 0.457 \\
\hline
\end{tabular}

CAP, community-acquired pneumonia; IQR, interquartile range; MRSA, methicillin-resistant Staphylococcus aureus; PCR, polymerase chain reaction; SC, serum creatinine. 
Table 2. Baseline Characteristics: MRSA PCR With Appropriate Utilization vs Inappropriate Utilization

\begin{tabular}{|c|c|c|c|}
\hline Characteristic & $\begin{array}{l}\text { Appropriate } \\
\text { Utilization } \\
(n=94)\end{array}$ & $\begin{array}{l}\text { Inappropriate } \\
\text { Utilization } \\
(n=61)\end{array}$ & $P$ Value \\
\hline Age, mean (SD), yr & $69.83(17.23)$ & $66.46(18.05)$ & 0.245 \\
\hline Race, no. (\%) & & & 0.634 \\
\hline White & $63(67.7)$ & $40(65.6)$ & \\
\hline Black & $10(10.8)$ & $9(14.8)$ & \\
\hline Asian & $3(3.2)$ & $0(0)$ & \\
\hline Hispanic & $12(12.9)$ & $8(13.1)$ & \\
\hline Other & $5(5.4)$ & $4(6.6)$ & \\
\hline Gender, no. (\%) & & & 0.780 \\
\hline Female & $41(43.6)$ & $28(45.9)$ & \\
\hline Male & $53(56.4)$ & $33(54.1)$ & \\
\hline Weight, mean (SD), kg & $78.26(20.97)$ & $80.86(23.19)$ & 0.470 \\
\hline Body mass index, mean (SD) & $28.02(7.22)$ & $28.70(7.13)$ & 0.564 \\
\hline Hospital, no. (\%) & & & 0.122 \\
\hline Hartford Hospital & $75(79.8)$ & $42(68.9)$ & \\
\hline Hospital of Central Connecticut & $19(20.2)$ & $19(31.1)$ & \\
\hline Charlson Comorbidity Index, median (IQR) & $5.0(4.0-7.0)$ & $5.0(3.0-7.0)$ & 0.590 \\
\hline Type of pneumonia, no. (\%) & & & 0.025 \\
\hline CAP & $28(29.8)$ & $19(31.1)$ & \\
\hline CAP with risk factors & $63(67.0)$ & $33(54.1)$ & \\
\hline Hospital-acquired pneumonia & $3(3.2)$ & $9(14.8)$ & \\
\hline Intensive care unit admission, no. (\%) & $18(19.1)$ & $17(27.9)$ & 0.205 \\
\hline Medical/surgical admission, no. (\%) & 77 (81.9) & $43(70.5)$ & 0.097 \\
\hline \multicolumn{4}{|l|}{ Anti-MRSA agent, no. (\%) } \\
\hline Vancomycin & 93 (98.9) & $61(100)$ & \\
\hline Linezolid & $1(1.1)$ & $0(0)$ & \\
\hline Chest radiograph positive, no. (\%) & $82(88.2)$ & $49(80.3)$ & 0.182 \\
\hline Infectious diseases consult, no. (\%) & $27(28.7)$ & $28(45.9)$ & 0.029 \\
\hline Pulmonary consult, no. (\%) & $33(35.1)$ & $20(32.8)$ & 0.766 \\
\hline Sepsis at time of anti-MRSA initiation, no. (\%) & $41(43.6)$ & 24 (39.3) & 0.598 \\
\hline Vasopressors at time of anti-MRSA initiation, no. (\%) & $2(2.1)$ & $4(6.6)$ & 0.163 \\
\hline $\mathrm{SC}$ at time of anti-MRSA initiation, median (IQR), mg/dL & $1.00(0.75-1.50)$ & $1.00(0.75-1.50)$ & 0.630 \\
\hline SC at time of anti-MRSA discontinuation, median (IQR), mg/dL & $1.00(0.70-1.40)$ & $0.90(0.70-1.40)$ & 0.679 \\
\hline SC $48 \mathrm{hr}$ after anti-MRSA discontinuation, median (IQR), mg/dL & $0.85(0.60-1.30)$ & $0.80(0.70-1.80)$ & 0.647 \\
\hline No. of nephrotoxic medications, excluding vancomycin, mean (SD) & $2.38(1.25)$ & $2.62(1.16)$ & 0.232 \\
\hline Use of intravenous contrast, no. (\%) & $39(41.5)$ & $25(41.0)$ & 0.950 \\
\hline \multicolumn{4}{|l|}{ Other antibiotics used, no. (\%) } \\
\hline Azithromycin & $41(43.6)$ & $22(36.1)$ & 0.350 \\
\hline Carbapenems & $4(4.3)$ & 2 (3.3) & 0.758 \\
\hline Cefepime & 82 (87.2) & $56(91.8)$ & 0.374 \\
\hline Ceftriaxone & $43(45.7)$ & $30(49.2)$ & 0.675 \\
\hline Piperacillin/tazobactam & $7(7.4)$ & $9(14.8)$ & 0.144 \\
\hline
\end{tabular}

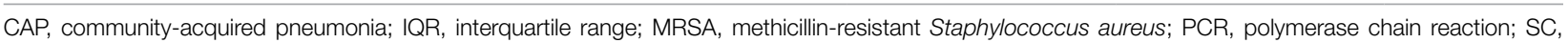
serum creatinine. 
Table 3. Primary and Secondary Outcomes: MRSA PCR vs No MRSA PCR Testing

\begin{tabular}{|c|c|c|c|}
\hline & $\begin{array}{c}\text { No MRSA PCR } \\
(n=164)\end{array}$ & $\begin{array}{c}\text { MRSA PCR } \\
(n=155)\end{array}$ & $P$ Value \\
\hline Days of therapy, median (IQR) & $1.86(0.56-3.33)$ & $1.68(0.80-2.74)$ & 0.458 \\
\hline Length of stay, median (IQR), days & $5.0(3.0-7.0)$ & $6.0(4.0-10.0)$ & 0.001 \\
\hline Readmission related to previous visit, no. (\%) & $14(8.5)$ & $15(9.7)$ & 0.723 \\
\hline Acute kidney injury, no. (\%) & $26(15.9)$ & $33(21.3)$ & 0.211 \\
\hline
\end{tabular}

exact test for $2 \times 2$ tables and a Pearson chi-square test for comparisons involving more than 2 groups.

Since anti-MRSA DOT (primary outcome) and LOS (secondary outcome) are often non-normally distributed, they have been transformed (eg, log or square root, again depending on distribution). Whichever native variable or transformation variable was appropriate was used as the outcome measure in a linear regression model to account for the influence of factors (covariates) that show significant univariate differences. Given the relatively small sample size, a maximum of 10 variables were included in the model. All factors were iterated in a forward regression model (most influential first) until no significant changes were observed.

All calculations were performed with SPSS v. 21 (IBM; Armonk, NY) using an a priori alpha level of 0.05 , such that all results yielding $P<0.05$ were deemed statistically significant.

\section{Results}

Of the 561 patient records reviewed, 319 patients were included and 242 patients were excluded. Reasons for exclusion included 65 patients admitted for a duration of 48 hours or less or discharged from the emergency department; 61 patients having another infection requiring anti-MRSA therapy; 60 patients not having a diagnosis of a LRTI or not receiving anti-MRSA therapy; 52 patients having received anti-MRSA therapy within 30 days prior to admission; and 4 patients treated outside of the specified date range.

Of the 319 patients included, 155 (48.6\%) were in the MRSA PCR group and 164 (51.4\%) were in the group that did not undergo MRSA PCR (Table 1). Of the 155 patients with a MRSA PCR ordered, the test was utilized appropriately in 94 (60.6\%) patients and inappropriately in 61 (39.4\%) patients (Table 2). In the MRSA PCR group, 135 patients had a negative result on PCR assay, with 133 of those patients having negative respiratory cultures, resulting in a NPV of $98.5 \%$. Differences in baseline characteristics between the MRSA PCR and no MRSA PCR groups were observed. The patients in the MRSA PCR group appeared to be significantly more ill than those in the no MRSA PCR group, as indicated by statistically significant differences in intensive care unit (ICU) admissions $(P=0.001)$, positive chest radiographs $(P=0.034)$, sepsis at time of anti-MRSA initiation ( $P=0.013)$, pulmonary consults placed $(P=0.003)$, and carbapenem usage $(P=0.047)$.

In the subgroup analysis comparing appropriate and inappropriate utilization within the MRSA PCR group, the inappropriate utilization group had significantly higher numbers of infectious diseases consults placed, patients with hospital-acquired pneumonia, and patients with community-acquired pneumonia with risk factors.

\section{Outcomes}

Median anti-MRSA DOT in the MRSA PCR group was shorter than DOT in the no MRSA PCR group, but this difference did not reach statistical significance (1.68 [IQR, 0.80-2.74] vs 1.86 days [IQR, 0.56-3.33], $P=0.458$;

Table 3). LOS in the MRSA PCR group was longer than in the no MRSA PCR group (6.0 [IQR, 4.0-10.0] vs 5.0 [IQR, 3.0-7.0] days, $P=0.001$ ). There was no difference in 30-day readmissions that were related to the previous visit or incidence of AKI between groups.

In the subgroup analysis, anti-MRSA DOT in the MRSA PCR group with appropriate utilization was shorter than DOT in the MRSA PCR group with inap- 


\begin{tabular}{|c|c|c|c|}
\hline & $\begin{array}{l}\text { Appropriate } \\
\text { Utilization } \\
\text { (n=94) }\end{array}$ & $\begin{array}{c}\text { Inappropriate } \\
\text { Utilization } \\
(n=61)\end{array}$ & $P$ Value \\
\hline Days of therapy, median (IQR) & $1.16(0.44-1.88)$ & $2.68(1.75-3.76)$ & $<0.001$ \\
\hline Length of stay, median (IQR), days & $5.0(4.0-7.0)$ & $7.0(5.0-12.0)$ & $<0.001$ \\
\hline Readmission related to previous visit, no. (\%) & $13(13.8)$ & $2(3.3)$ & 0.030 \\
\hline Acute kidney injury, no. (\%) & $17(18.1)$ & $16(26.2)$ & 0.226 \\
\hline
\end{tabular}

propriate utilization (1.16 [IQR, 0.44-1.88] vs 2.68 [IQR, 1.75-3.76] days, $P<0.001$; Table 4). LOS in the MRSA PCR group with appropriate utilization was shorter than LOS in the inappropriate utilization group (5.0 [IQR, 4.0-7.0] vs 7.0 [IQR, 5.0-12.0] days, $P<0.001$ ). Thirtyday readmissions that were related to the previous visit were significantly higher in patients in the MRSA PCR group with appropriate utilization (13 vs $2, P=0.030$ ). There was no difference in incidence of AKI between the groups.

A multivariate analysis was completed to determine whether the sicker MRSA PCR population was confounding outcomes, particularly the secondary outcome of LOS, which was noted to be longer in the MRSA PCR group (Table 5). When comparing LOS in the MRSA PCR and the no MRSA PCR patients, the multivariate analysis showed that admission to the ICU and carbapenem use were associated with a longer LOS $(P<0.001$ and $P=0.009$, respectively). The incidence of admission to the ICU and carbapenem use were higher in the MRSA PCR group $(P=0.001$ and $P=0.047)$. Therefore, longer LOS in the MRSA PCR patients could be a result of the higher prevalence of ICU admissions and infections requiring carbapenem therapy rather than the result of the MRSA PCR itself.

\section{Discussion}

A MRSA PCR nasal swab protocol can be used to minimize a patient's exposure to unnecessary broadspectrum antibiotics, thereby preventing antimicrobial resistance. Thus, it is important to assess how our health system is utilizing this antimicrobial stewardship tactic. With the MRSA PCR's high NPV, providers can be confident that MRSA pneumonia is unlikely in the absence of MRSA colonization. Our study established a NPV of $98.5 \%$, which is similar to other studies, all of which have shown NPVs greater than 95\%..$^{5-8}$ Despite the high NPV, this study demonstrated that only $51.4 \%$ of patients with LRTI had orders for a MRSA PCR. Of the 155 patients with a MRSA PCR, the test was utilized appropriately only $60.6 \%$ of the time. A majority of the inappropriately utilized tests were due to MRSA PCR orders placed more than 48 hours after anti-MRSA therapy initiation. To our knowledge, no other studies have assessed the clinical utility of MRSA PCR nasal swabs as an antimicrobial stewardship tool in a diverse health system; therefore, these results are useful to guide future practices at our institution. There is a clear need for provider and pharmacist education to increase the use of MRSA PCR nasal swab testing for patients with LRTI being treated with anti-MRSA therapy. Additionally, clinician education regarding the initial timing of the MRSA PCR order and the proper utilization of the results of the MRSA PCR likely will benefit patient outcomes at our institution.

When evaluating anti-MRSA DOT, this study demonstrated a reduction of only 0.18 days (about 4 hours) of anti-MRSA therapy in the patients who received MRSA PCR testing compared to the patients without a MRSA PCR ordered. Our anti-MRSA DOT reduction was lower than what has been reported in similar studies. For example, Baby et al found that the use of the MRSA PCR was associated with 46.6 fewer hours of unnecessary antimicrobial treatment. Willis et al evaluated a pharmacist-driven protocol that resulted in a reduction of 1.8 days of anti-MRSA therapy, despite a protocol 
Table 5. Multivariate Analyses

\begin{tabular}{|c|c|c|c|c|c|c|}
\hline Outcome Affected & Variable & $\beta$ & Std $\beta$ & LB $\beta$ & UB $\beta$ & $P$ Value \\
\hline \multicolumn{7}{|c|}{ MRSA PCR vs No MRSA PCR } \\
\hline \multirow{3}{*}{ Length of stay (log) } & ICU admission & 4.281 & 0.233 & 2.426 & 6.137 & $<0.001$ \\
\hline & Days of therapy & 0.786 & 0.218 & 0.426 & 1.147 & $<0.001$ \\
\hline & Carbapenem use & 6.124 & 0.137 & 1.544 & 10.704 & 0.009 \\
\hline \multicolumn{7}{|c|}{ MRSA PCR with Appropriate vs Inappropriate Utilization } \\
\hline \multirow{2}{*}{ Days of therapy (log) } & Appropriate utilization & -0.401 & -0.371 & -0.560 & -0.243 & $<0.001$ \\
\hline & Vancomycin trough $>20 \mathrm{mcg} / \mathrm{mL}$ & 0.334 & 0.181 & 0.064 & 0.604 & 0.016 \\
\hline \multirow{2}{*}{ Length of stay (log) } & Infectious diseases consult & 0.156 & 0.279 & 0.072 & 0.240 & $<0.001$ \\
\hline & Appropriate utilization & -0.126 & -0.229 & -0.208 & -0.044 & 0.003 \\
\hline
\end{tabular}

ICU, intensive care unit; LB, lower bound; MRSA, methicillin-resistant Staphylococcus aureus; PCR, polymerase chain reaction; Std $\beta$, standardized beta; UB, upper bound.

compliance rate of only $55 \% .^{9,10}$ In our study, the patients in the MRSA PCR group appeared to be significantly more ill than those in the no MRSA PCR group, which may be the reason for the incongruences in our results compared to the current literature. Characteristics such as ICU admissions, positive chest radiographs, sepsis cases, pulmonary consults, and carbapenem usageall of which are indicative of a sicker population-were more prevalent in the MRSA PCR group. This sicker population could have underestimated the reduction of DOT in the MRSA PCR group compared to the no MRSA PCR group.

After isolating the MRSA PCR patients in the subgroup analysis, anti-MRSA DOT was 1.5 days shorter when the test was appropriately utilized, which is more comparable to what has been reported in the literature..$^{9,10}$ Only $60.6 \%$ of the MRSA PCR patients had their anti-MRSA therapy appropriately managed based on the MRSA PCR. Interestingly, a majority of patients in the inappropriate utilization group had MRSA PCR tests ordered more than 48 hours after beginning antiMRSA therapy. More prompt and efficient ordering of the MRSA PCR may have resulted in more opportunities for earlier de-escalation of therapy. Due to these factors, the patients in the inappropriate utilization group could have further contributed to the underestimated difference in anti-MRSA DOT between the MRSA PCR and no MRSA PCR patients in the primary outcome. Additionally, there were no notable differences between the appropriate and inappropriate utilization groups, unlike in the MRSA PCR and no MRSA PCR groups, which is why we were able to draw more robust conclusions in the subgroup analysis. Therefore, the subgroup analysis confirmed that if the results of the MRSA PCR are used appropriately to guide anti-MRSA therapy, patients can potentially avoid 36 hours of broad-spectrum antibiotics.

Data on how the utilization of the MRSA PCR nasal swab can affect LOS are limited; however, one study did report a 2.8-day reduction in LOS after implementation of a pharmacist-driven MRSA PCR nasal swab protocol.11 Our study demonstrated that LOS was significantly longer in the MRSA PCR group than in the no MRSA PCR group. This result was likely affected by the aforementioned sicker MRSA PCR population. Our multivariate analysis further confirmed that ICU admissions were associated with a longer LOS, and, given that the MRSA PCR group had a significantly higher ICU population, this likely confounded these results. If our 2 groups had had more evenly distributed characteristics, it is possible that we could have found a shorter LOS in the MRSA PCR group, similar to what is reported in the literature. In the subgroup analysis, LOS was 2 days shorter in the appropriate utilization group compared to the inappropriate utilization group. This further affirms that the results of the MRSA PCR must be used appropriately in order for patient outcomes, like LOS, to benefit. 
The effects of the MRSA PCR nasal swab on 30-day readmission rates and incidence of $A K I$ are not well-documented in the literature. One study did report 30-day readmission rates as an outcome, but did not cite any difference after the implementation of a protocol that utilized MRSA PCR nasal swab testing. ${ }^{12}$ The outcome of AKI is slightly better represented in the literature, but the results are conflicting. Some studies report no difference after the implementation of a MRSA PCR-based protocol, ${ }^{11}$ and others report a significant decrease in AKI with the use of the MRSA PCR. ${ }^{9}$ Our study detected no difference in 30-day readmission rates related to the previous admission or in AKI between the MRSA PCR and no MRSA PCR populations. In the subgroup analysis, 30-day readmission rates were significantly higher in the MRSA PCR group with appropriate utilization than in the group with inappropriate utilization; however, our study was not powered to detect a difference in this secondary outcome.

This study had some limitations that may have affected our results. First, this study was a retrospective chart review. Additionally, the baseline characteristics were not well balanced across the different groups. There were sicker patients in the MRSA PCR group, which may have led to an underestimate of the reduction in DOT and LOS in these patients. Finally, we did not include enough patient records to reach power in the MRSA PCR group due to a higher than expected number of patients meeting exclusion criteria. Had we attained sufficient power, there may have been more profound reductions in DOT and LOS.

\section{Conclusion}

MRSA infections are a common cause for hospitalization, and there is a growing need for antimicrobial stewardship efforts to limit unnecessary antibiotic usage in order to prevent resistance. As illustrated in our study, appropriate utilization of the MRSA PCR can reduce DOT up to 1.5 days. However, our results suggest that there is room for provider and pharmacist education to increase the use of MRSA PCR nasal swab testing in patients with $L R T I$ receiving anti-MRSA therapy. Further emphasis on the appropriate utilization of the MRSA PCR within our health care system is essential.

Corresponding author: Casey Dempsey, PharmD, BCIDP, 80 Seymour St., Hartford, CT 06106; casey.dempsey@hhchealth.org.

Financial disclosures: None.

doi:10.12788/jcom.0020

\section{References}

1. Antimicrobial resistance threats. Centers for Disease Control and Prevention web site. www.cdc.gov/drugresistance/biggest-threats. html. Accessed September 9, 2020.

2. Biggest threats and data. Centers for Disease Control and Prevention web site. www.cdc.gov/drugresistance/biggest_threats. html\#mrsa. Accessed September 9, 2020.

3. Smith MN, Erdman MJ, Ferreira JA, et al. Clinical utility of methicillin-resistant Staphylococcus aureus nasal polymerase chain reaction assay in critically ill patients with nosocomial pneumonia. J Crit Care. 2017;38:168-171.

4. Giancola SE, Nguyen AT, Le B, et al. Clinical utility of a nasal swab methicillin-resistant Staphylococcus aureus polymerase chain reaction test in intensive and intermediate care unit patients with pneumonia. Diagn Microbiol Infect Dis. 2016;86:307-310.

5. Dangerfield B, Chung A, Webb B, Seville MT. Predictive value of methicillin-resistant Staphylococcus aureus (MRSA) nasal swab PCR assay for MRSA pneumonia. Antimicrob Agents Chemother. 2014;58:859-864.

6. Johnson JA, Wright ME, Sheperd LA, et al. Nasal methicillin-resistant Staphylococcus aureus polymerase chain reaction: a potential use in guiding antibiotic therapy for pneumonia. Perm J. 2015;19: 34-36.

7. Dureau AF, Duclos G, Antonini F, et al. Rapid diagnostic test and use of antibiotic against methicillin-resistant Staphylococcus aureus in adult intensive care unit. Eur J Clin Microbiol Infect Dis. 2017;36:267-272.

8. Tilahun B, Faust AC, McCorstin P, Ortegon A. Nasal colonization and lower respiratory tract infections with methicillin-resistant Staphylococcus aureus. Am J Crit Care. 2015;24:8-12.

9. Baby N, Faust AC, Smith T, et al. Nasal methicillin-resistant Staphylococcus aureus (MRSA) PCR testing reduces the duration of MRSA-targeted therapy in patients with suspected MRSA pneumonia. Antimicrob Agents Chemother. 2017;61:e02432-16.

10. Willis C, Allen B, Tucker C, et al. Impact of a pharmacist-driven methicillin-resistant Staphylococcus aureus surveillance protocol. Am J Health-Syst Pharm. 2017;74:1765-1773.

11. Dadzie P, Dietrich T, Ashurst J. Impact of a pharmacist-driven methicillin-resistant Staphylococcus aureus polymerase chain reaction nasal swab protocol on the de-escalation of empiric vancomycin in patients with pneumonia in a rural healthcare setting. Cureus. 2019;11:e6378

12. Dunaway S, Orwig KW, Arbogast ZQ, et al. Evaluation of a pharmacy-driven methicillin-resistant Staphylococcus aureus surveillance protocol in pneumonia. Int J Clin Pharm. 2018;40;526-532. 